\title{
haemodynamics in patients with pseudoexfoliation glaucoma
}

Eye (2005) 19, 1297-1300. doi:10.1038/sj.eye.6701776; published online 14 January 2005

Keywords: pseudoexfoliation glaucoma; cerebral haemodynamics; transcranial colour Doppler

\section{Introduction}

Pseudoexfoliation syndrome characterized by the appearance of dandruff-like flakes and whitish granular deposits on the lens and on the pupillary border is a systemic disease, including orbital tissue, skin, lung, heart, liver, gall bladder, kidney, ear, optic nerve, blood vessels, and meninges. ${ }^{1-5}$ Pseudoexfoliation is the most common precursor of open-angle glaucoma. ${ }^{6}$ It has been shown that pseudoexfoliation syndrome is associated with an alteration in the retrobulbar haemodynamics. ${ }^{7}$ This alteration is more prominent in pseudoexfoliation glaucoma. ${ }^{7}$

A recent study has shown that total cerebral blood perfusion is reduced and the cerebrovascular responses to hyperoxia are abnormal in open-angle glaucoma patients compared with controls. ${ }^{8}$ Pseudoexfoliation is a systemic disorder ${ }^{1-3}$ and has an association with the vascular diseases, including angina, acute myocardial infarction, or stroke. ${ }^{9}$ In addition, a high frequency of pseudoexfoliation has indeed been reported in patients with transient ischaemic attacks. ${ }^{10}$ Thus, we hypothesized that pseudoexfoliation glaucoma might have an alteration in the blood flow velocity and resistance measured in the middle cerebral artery (MCA).

Transcranial colour Doppler (TCD) method for the measurement of intracranial arterial blood flow velocity is a safe and noninvasive technique. The good reproducibility of TCD
${ }^{1}$ Department of Ophthalmology, University of Kirikkale, 71100 Kirikkale, Turkey

${ }^{2}$ Department of Radiology, University of Kirikkale, 71100 Kirikkale, Turkey

Correspondence: C Akarsu, Cagdas Sokak 37/13, TR 06130, Aydinlikevler, Ankara, Turkey Tel: + 903182252820 ; Fax: +90 3182252819 . E-mail: cengizakarsu@ hotmail.com

Received: 30 June 2004 Accepted: 20 October 2004 Published online: 14 January 2005

The authors have no commercial or proprietary interest in any of the equipment and medications mentioned in this study.

This study in part was presented as a poster in the 24th National Radiology Congress 8-11 October 2003, Ankara, Turkey. 
velocity measurements in the MCA has been shown in previous studies. ${ }^{11,12}$ TCD gives information about flow velocities. The measurements cannot be used as a direct measure of bulk blood flow. However, there is a good correlation between blood flow velocity and bulk blood flow in the cerebral arteries. ${ }^{13,14}$

In the current study, we evaluated the cerebral haemodynamics in patients with pseudoexfoliation glaucoma using TCD and compared with those of the healthy controls.

\section{Materials and methods}

Consecutive patients with pseudoexfoliation glaucoma were identified from the Out-patients Department of Ophthalmology of the University of Kirikkale, and they were invited to participate in this study. A total of 19 patients with pseudoexfoliation glaucoma and 19 ageand sex-matched healthy volunteers were enrolled. All participants gave informed consent to the study and the tenets of the Declaration of Helsinki were followed.

All subjects underwent complete ophthalmic examination, including visual acuity test, biomicroscopy, tonometry, gonioscopy, and fundoscopic examination. The diagnosis of pseudoexfoliation glaucoma was based on the presence of dust-like material on the lens and/or pupillary border, increased pigmentation at the angle, glaucomatous optic disc change, and glaucomatous visual field defects on the 30-2 Humphrey Full Threshold Test (Humphrey Instruments, San Leandro, CA, USA). Of 19 glaucoma patients, nine were on prostaglandin analogues, six were on timolol, and four were untreated. Control subjects had no history of ophthalmic problem and had normal ophthalmic examination. All

participants had a best-corrected visual acuity of 20/30 or better, and they had no history of cardiovascular or respiratory diseases, arterial hypertension, diabetes, and migraine. Excluded from the study were patients with eye disease other than pseudoexfoliation glaucoma, transient ischaemic attacks, any neurological disorder or oral contraceptive usage. Pregnant or nursing women and smokers were also excluded from the study. Before the evaluation of the cerebral haemodynamics, the carotid artery was evaluated in each case. In case of abnormal circulation or obstruction in the carotid artery, this case was excluded from the study.

In each patient with pseudoexfoliation glaucoma, the affected eye in patient with unilateral glaucoma and the eye with more advanced lesions in patient with bilateral glaucoma were included in the study, and the ipsilateral MCAs were evaluated. In the control subjects, the eye and the ipsilateral MCA were chosen randomly.

At a prestudy visit, intraocular pressure (IOP)lowering medications were discontinued. Required washout periods were 4 weeks for $\beta$-blockers and prostaglandin analogues. After the washout period, glaucoma patients returned for the study. A masked observer measured IOP by Goldmann applanation tonometer, systemic blood pressure by cuff, and heart rate by palpation of radial pulse.

TCD examinations were performed at the Department of Radiology by a masked sonographer using lineararray real-time ultrasound with a $2-5 \mathrm{MHz}$ multifrequency sector probe (GE Logiq 9 Milwaukee, WI, USA). The MCA was examined through the transtemporal window at an examination depth approximately $50-65 \mathrm{~mm}$ while the participants comfortably reclined in a supine position. The Doppler sample volume cursor was aligned along the colour flow image of the vessels, the optimum insonation angle (maximum $45^{\circ}$ ) was applied, and when the highest velocity measurements were obtained, spectral waveforms were recorded. The peak systolic velocity (PSV), end-diastolic velocity (EDV), time-averaged maximum velocity (TAMAX), resistivity index $(\mathrm{RI}=[\mathrm{PSV}-\mathrm{EDV}] / \mathrm{PSV})$, and pulsatility index $(\mathrm{PI}=[\mathrm{PSV}-\mathrm{EDV}] /$ mean velocity $)$ were measured automatically by the colour Doppler unit. The results were expressed as mean \pm standard deviation.

The minimum sample size to detect the differences on the middle cerebral artery blood flow velocity by means of this technique was calculated on the basis of previous study. ${ }^{8}$ A sample size of 19 was chosen to provide $80 \%$ power to detect a $20 \%$ change in flow velocity or pulsatility index in the MCA. Statistical analysis was performed using the unpaired Student's $t$-test and $\chi^{2}$-test for the comparison between glaucoma patients and controls. Differences at a level of $P<0.05$ were accepted as statistically significant changes.

\section{Results}

The general characteristics of the subjects are summarized in Table 1 . There was no significant difference in mean age $(P=0.36)$, gender $(P=1.00)$ mean blood pressure $(P=0.19$ for systolic blood pressure and $P=0.91$ for diastolic blood pressure), and mean pulse rate $(P=0.06)$ between the groups. Each patient with pseudoexfoliation glaucoma had an IOP of $>21 \mathrm{mmHg}$ after the washout period. The mean IOP in the pseudoexfoliation glaucoma patients was significantly higher than in the controls $(P<0.001)$.

The haemodynamic parameters of the MCA are represented in Table 2. The mean PSV, EDV, and TAMAX were significantly lower in the pseudoexfoliation glaucoma patients than in the controls $(P<0.001$, $<0.001$ and $<0.001$, respectively). Similarly, the pseudoexfoliation glaucoma patients had significantly 
Table 1 Subjects' characteristics, blood pressure, heart rate, and IOP

\begin{tabular}{lccc}
\hline Variables & Pseudoexfoliation (mean \pm SD) & Control (mean \pm SD) & P-value (t-test) \\
\hline Age (years) & $60.0 \pm 6.2$ & $57.3 \pm 10.7$ & 0.36 \\
Gender (male:female) & $6: 13$ & $6: 13$ & 1.00 \\
Systolic blood pressure (mmHg) & $120.0 \pm 8.9$ & $123.9 \pm 9.2$ & 0.19 \\
Diastolic blood pressure (mmHg) & $80.5 \pm 6.2$ & $79.7 \pm 7.4$ & 0.91 \\
Heart rate (beats/min) & $62.9 \pm 5.9$ & $66.5 \pm 5.4$ & 0.06 \\
IOP (mmHg) & $24.5 \pm 2.4$ & $15.6 \pm 2.9$ & $<0.001$ \\
\hline
\end{tabular}

Table 2 Haemodynamic parameters measured in the middle cerebral artery

\begin{tabular}{lccc}
\hline Parameters & $\begin{array}{c}\text { Pseudoexfoliation } \\
(\text { mean } \pm \text { SD })\end{array}$ & $\begin{array}{c}\text { Control } \\
(\text { mean } \pm \text { SD })\end{array}$ & $\begin{array}{c}\text { P-value } \\
(\text { t-test })\end{array}$ \\
\hline PSV (cm/s) & $57.1 \pm 11.3$ & $81.3 \pm 12.2$ & $<0.001$ \\
EDV (cm/s) & $22.8 \pm 6.8$ & $38.5 \pm 9.1$ & $<0.001$ \\
PI & $0.98 \pm 0.21$ & $0.79 \pm 0.12$ & 0.001 \\
RI & $0.58 \pm 0.10$ & $0.53 \pm 0.07$ & 0.04 \\
TAMAX & $36.4 \pm 9.3$ & $55.9 \pm 14.3$ & $<0.001$ \\
\hline
\end{tabular}

PSV, peak systolic velocity; EDV, end-diastolic velocity; TAMAX, timeaveraged maximum velocity; RI, resistance index; PI, pulsatility index.

higher PI and RI than did the controls $(P=0.001$ and 0.04 , respectively).

\section{Discussion}

The current study revealed that cerebral haemodynamics in patients with pseudoexfoliation glaucoma was altered in comparison to controls. Our findings confirmed the previous TCD study suggesting reduced total cerebral perfusion in open-angle glaucoma patients (high tension and normal tension). ${ }^{8}$ The results of this study also support the magnetic resonance imaging studies, which have shown diffuse cerebral ischaemic changes, ${ }^{15}$ cerebral infarcts and corpus callosum atrophy in glaucoma patients. ${ }^{16}$

Pseudoexfoliation glaucoma is also associated with the alterations in the retrobulbar blood flow parameters. ${ }^{7}$ Similar alterations in the retrobulbar haemodynamics have been found in various types of glaucoma. ${ }^{17,18}$ Like retrobulbar circulation, ${ }^{19}$ these cerebral blood flow alterations found in the current and previous studies suggest that insufficient cerebral blood flow is associated with glaucoma. However, it is beyond the scope of the current study whether these alterations contribute to the pathophysiology of pseudoexfoliation glaucoma or they are simply a consequence of the disease.

Pseudoexfoliation is a progressive process, that is to say; the amount of the pseudoexfoliative material is correlated with the clinical findings. ${ }^{20}$ Pseudoexfoliative material deposits in the vascular endothelial cells, smooth muscle cells and pericytes. ${ }^{21,22}$ In addition, pseudoexfoliation has been shown to be associated with elastosis in the vascular wall. ${ }^{23,24}$ Since elastin is one of the major components of the extracellular matrix of arteries; abnormal elastic fibre and elastin synthesis in pseudoexfoliation may be associated with vascular disease or vascular risk factors. ${ }^{9}$ Although there has been no findings that major vessels are significantly affected, smaller vessels and microcirculation are markedly involved in pseudoexfoliation glaucoma. ${ }^{3}$ If ocular pseudoexfoliation is a finding of widespread distribution of pseudoexfoliative materials, the deposit of these materials in the wall of the MCA may explain the reduction in blood flow velocity and elevation in the resistance in the cerebral circulation.

This was a controlled study for factors which might affect the cerebral blood flow velocity such as age, sex, and systemic blood pressure. Therefore, we hoped to eliminate the effect of these variables and to give a more useful comparison between pseudoexfoliation glaucoma patients and healthy controls. It may be argued that the alteration in the cerebral circulation is because of the increased IOP in the pseudoexfoliation glaucoma patients, or, alternatively it is due to open-angle glaucoma mechanism. ${ }^{8}$ It is unclear whether IOP elevation can interfere with the cerebral blood flow velocities. On the other hand, in a group of ocular hypertension patients, we revealed that cerebral circulation was not affected despite the higher IOP. ${ }^{25}$ Further studies will clarify the effects of IOP on the cerebral haemodynamics.

In conclusion, this study suggests that cerebral blood flow in pseudoexfoliation glaucoma is altered compared with controls. Our results support the hypothesis that there is a vascular insufficiency at least in some pseudoexfoliation glaucoma patients. Further studies are required to provide direct evidence for the vascular changes observed in pseudoexfoliation glaucoma.

\section{References}

1 Naumann GOH, Schlőtzer-Schrehardt U, Küchle M. Pseudoexfoliation syndrome for comprehensive ophthalmologist. Intraocular and systemic manifestations. Ophthalmology 1998; 105: 951-968. 
2 Schlőtzer-Schrehardt U, Koca MR, Naumann GOH, Volkholz H. Pseudoexfoliation syndrome. Ocular manifestation of a systemic disorder? Arch Ophthalmol 1992; 110: 1752-1756.

3 Streeten BW, Li ZY, Wallace RN, Eagle Jr RC, Keshgegian AA. Pseudoexfoliative fibrillopathy in visceral organs of a patient with pseudoexfoliation syndrome. Arch Ophthalmol 1992; 110: 1757-1762.

4 Streeten BW, Dark AJ, Wallace RN, Li ZY, Hoepner JA. Pseudoexfoliative fibrillopathy in the skin of patients with ocular pseudoexfoliation. Am J Ophthalmol 1990; 110: 490-499.

5 Cahill M, Early A, Stack S, Blayney AW, Eustace P. Pseudoexfoliation and sensorineural hearing loss. Eye 2002; 16: 261-266.

6 Ritch R. Exfoliation syndrome-the most common identifiable cause of open angle-glaucoma. J Glaucoma 1994; 3: $176-178$.

7 Yüksel N, Karabaş L, Arslan A, Demirci A, Çaglar Y. Ocular hemodynamics in pseudoexfoliation syndrome and pseudoexfoliation glaucoma. Ophthalmology 2001; 108: 1043-1049.

8 Harris A, Zarfati D, Zalish M, Biller J, Sheets CW, Rechtman E et al. Reduced cerebrovascular blood flow velocities and vasoreactivity in open-angle glaucoma. Am J Ophthalmol 2003; 135: 144-147.

9 Mitchell P, Wang JJ, Smith W. Association of pseudoexfoliation syndrome with increased vascular risk. Am J Ophthalmol 1997; 124: 685-687.

10 Repo LP, Suhonen MT, Terasvirta ME, Koivisto KJ. Color Doppler imaging of the ophthalmic artery blood flow spectra of patients who have had a transient ischemic attack. Correlations with generalized iris transluminance and pseudoexfoliation syndrome. Ophthalmology 1995; 102: 1199-1205.

11 Baumgartner RW, Mathis J, Sturzenegger M, Mattle HP. A validation study on the intraobserver reproducibility of transcranial color-coded duplex sonography velocity measurements. Ultrasound Med Biol 1994; 20: 233-237.

12 Maeda H, Etani H, Handa N, Mattle HP. A validation study on the reproducibility of transcranial Doppler velocimetry. Ultrasound Med Biol 1990; 16: 9-14.

13 Taylor GA, Short BL, Walker LK, Traystman RJ. Intracranial blood flow: quantification with duplex Doppler and color Doppler flow US. Radiology 1990; 176: 231-236.
14 Hansen NB, Stonestreet BS, Rosenkrantz TS, Oh W. Validity of Doppler measurements of anterior cerebral artery blood flow velocity: correlation with brain blood flow in piglets. Pediatrics 1983; 72: 526-531.

15 Stroman GA, Stewart WC, Golnik KC, Cure JK, Olinger RE. Magnetic resonance imaging in patients with low-tension glaucoma. Arch Ophthalmol 1995; 113: $168-172$.

16 Ong K, Farinelli A, Billson F, Houang M, Stern M. Comparative study of brain magnetic resonance imaging findings in patients with low-tension glaucoma and control subjects. Ophthalmology 1995; 102: 1632-1638.

17 Akarsu C, Bilgili MYK. Color Doppler imaging in ocular hypertension and open-angle glaucoma. Graefes Arch Clin Exp Ophthalmol 2004; 242: 125-129.

18 Kaiser HJ, Schoetzau A, Stumpfig D, Flammer J. Blood-flow velocities of the extraocular vessels in patients with hightension and normal-tension primary open-angle glaucoma. Am J Ophthalmol 1997; 123: 320-327.

19 Schumann J, Orgul S, Gugleta K, Dubler B, Flammer J. Interocular difference in progression of glaucoma correlates with interocular differences in retrobulbar circulation. Am J Ophthalmol 2000; 129: 728-733.

20 Gottanka J, Flügel-Koch C, Martus P, Johnson DH, LutjenDrecoll E. Correlation of pseudoexfoliative material and optic nerve damage in pseudoexfoliation syndrome. Invest Opthalmol Vis Sci 1997; 38: 2435-2446.

21 Anderson DR, Davis EB. Glaucoma, capillaries and pericytes: 5 . Preliminary evidence that carbon dioxide relaxes pericyte contractile tone. Ophthalmologica 1996; 210: $280-284$

22 Konstas AGP, Marshall GE, Cameron SA, Lee WR. Morphology of iris vasculopathy in exfoliation glaucoma. Acta Ophthalmol (Copenh) 1993; 71: 751-759.

23 Konstas AGP, Marshall GE, Lee WR. Immunogold localisation of laminin in normal and exfoliative iris. Br J Ophthalmol 1990; 74: 450-457.

24 Streeten BW, Bookman L, Ritch R, Prince AM, Dark AJ. Pseudoexfoliative fibrillopathy in the conjunctiva: a relation to elastic fibers and elastosis. Ophthalmology 1987; 94: 1439-1449.

25 Akarsu C, Karadeniz-Bilgili Y, Unal B, Taner P, Ergin A, Altan-Kara S. Cerebral hemodynamics in ocular hypertension. Graefes Arch Clin Exp Ophthalmol, DOI:10.1007/s00417-004-0963-2. 